\title{
Poor Outcome and Prolonged Hospital Stay after the Diagnosis of Liver Abscesses in Hospitalized Cancer Patients: A Single Center Experience
}

\begin{abstract}
Parham Khosravi-Shahi, María Inmaculada, Aparicio-Salcedo, Manuel, Alva-Bianchi, Marta, Arregui-Valles, Blanca Isabel, Morón-García, Victoria Clara, Tirado-Anula, María, De Toro-Carmena, Íñigo, Martínez-Delfrade and Ricardo, González-del-Val
\end{abstract}

Servicio de Oncología Médica, Hospital General Universitario Gregorio Marañón, Spain

Submission: December 10, 2019; Published: January 23, 2020

"Correspondence Author: Parham khosravi-shahi, Servicio de Oncología Médica, Hospital General Universitario Gregorio Marañón. Doctor Esquerdo Street, 46. 28007, Madrid, Spain

Abstract

Background: Liver abscesses are uncommon, but they are associated with a hospital mortality rate of $10 \%$ in patients without cancer. However, its relevance in cancer patients in terms of survival and length of hospitalization is unknown.

Patients and Methods: We conducted an observational study in order to analyze the impact of liver abscesses in hospitalized cancer patients. Eligible patients were adults with a solid tumor, received antineoplastic treatment and were hospitalized in our department with a diagnosis of liver abscesses. Primary end-points of the study were overall survival (OS) and mean hospital stay (MHS). Secondary end-points were the description of risk factors of liver abscesses, isolated microorganisms and the specific treatment.

Results: In our study the incidence of liver abscesses in cancer patients was $1.85 \%$. Median age was 64 years, $60 \%$ were men, and the vast majority of patients had an advanced bilio-pancreatic tumor (80\%). With a median follow-up of 18 months, median OS was 6 months (95\% CI: 1-12); and OS probability at 18 -month was $27 \%$. MHS was 30.5 days (95\% CI: 20.3-40.7). Risk factors for developing liver abscesses were: a) bilio-pancreatic cancers; b) chemotherapy administration; c) severe malnutrition; and d) biliary drainage/prosthesis. Eight patients (53.3\%) had bacteremia, and the most common isolated microorganism was E.coli (33.3\%). Percutaneous drainage of abscesses was performed in $53.3 \%$ of the patients, and all patients were treated with broad-spectrum antibiotics.

Conclusion: Although liver abscesses were uncommon, they were associated with a prolonged hospitalization and poor outcome in cancer patients.

Keywords: Hepatic; Escherichia; Meropenem; Bacteremia

\section{Introduction}

Liver abscesses are uncommon, but they are associated with an overall hospital mortality rate of $6-10 \%$ in patients without cancer [1]. However, the incidence of liver abscess and its relevance in cancer patients in terms of survival and length of hospital stay is unknown. Cancer itself, complications related to cancer (such as malnutrition and tumor cachexia) and adverse events related to the cancer treatment, especially cytotoxic chemotherapy, are associated with immunosuppression and a higher risk of infection and bacteremia. In the literature, several case reports of liver abscesses in cancer patients have been published in last decades, and some cases were associated with poor prognosis. We hypothesized that liver abscesses could be associated with worse prognosis and longer hospital stay in cancer patients. Hence, we conducted a descriptive observational study in order to analyze the relevance of the presence of liver abscesses in hospitalized patients with solid Tumors [2].

\section{Patients and Methods}

\section{Study Design and Eligibility Criteria of Patients}

We conducted a prospective observational longitudinal study in order to analyze the relevance and the impact of liver abscesses diagnosis in cancer patients who were hospitalized in our Medical Oncology Department. In this study, we prospectively included all hospitalized cancer patients with a definitive liver 


\section{Cancer Therapy \& Oncology International Journal}

abscesses diagnosis, determined by imaging techniques, such as abdominal sonography, abdominal computed tomography (CT scan) and/or liver magnetic resonance, with or without microorganism isolation. Eligible patients were at least 18 years of age, had a histologically confirmed solid tumor, received an antineoplastic treatment (chemotherapy, immunotherapy or other type of therapy) and were hospitalized in our Medical Oncology Department between January and December of 2018. We excluded from this observational study all hospitalized cancer patients included in clinical trials. Hence, we included in this study all consecutive cases of cancer patients with liver abscesses, who had all eligibility criteria and provided informed consent.

\section{Study end-points}

Primary end-points of the study were overall survival (OS) and the length of hospitalization (mean hospital stay) associated with the diagnosis of liver abscesses in cancer patients who were hospitalized in our center. OS in this study was defined as the time from the diagnosis of liver abscesses until death from any cause. Secondary end-points of the study were the description of the risk factors associated with liver abscesses, the isolated microorganisms and the specific treatments used for the liver abscesses in this subgroup of patients.

\section{Statistical Analysis}

Standard summary statistics for continuous variables were median, range, and 95\% confidence interval (95\% CI). Standard summary statistics for discrete variables were count and proportion. Survival estimation (OS) was calculated with KaplanMeier methods, and expressed as median OS in months (with 95\% CI) and 12-month and 18-month OS probability (percentage). The stata statistical program, version 14 (StataCorp. 2007. Stata Statistical Software: Release 14. College Station, TX: StataCorp LP) was used for all statistical analyses.

\section{Results}

\section{Baseline characteristics of the study patients}

Between January and December of 2018, we prospectively identified 15 consecutive cases of liver abscesses in hospitalized cancer patients in our center, which resulted in an incidence of liver abscesses in this group of patients of $1.85 \%$ (15/810). Baseline clinical and pathological characteristics of the study population are detailed in table 1 . Median age of the patients was 64 years (range: $37-79), 60 \%$ (9/15) of the patients were men, $80 \%(12 / 15)$ of the patients had a bilio-pancreatic cancer and the vast majority of patients had a metastatic tumor $(93.3 \%$ [14/15] of patients had stage IV).

Table 1: Baseline clinical and pathological characteristics of the study population $(n=15)$.

\begin{tabular}{|c|c|}
\hline \multicolumn{2}{|c|}{ Baseline Characteristics } \\
\hline \multicolumn{2}{|c|}{ Age } \\
\hline Median (range) & 64 years $(37-79)$ \\
\hline$\geq 65$ years, $\mathrm{n}(\%)$ & $6(40 \%)$ \\
\hline$<65$ years, $\mathrm{n}(\%)$ & $9(60 \%)$ \\
\hline \multicolumn{2}{|c|}{ Gender } \\
\hline Male sex, n (\%) & $9(60 \%)$ \\
\hline Female sex, n (\%) & $6(40 \%)$ \\
\hline \multicolumn{2}{|c|}{ Histology and Primary Tumor } \\
\hline Pancreatic ductal adenocarcinoma, n (\%) & $8(53.3 \%)$ \\
\hline Cholangiocarcinoma, n (\%) & $3(20 \%)$ \\
\hline Gallbladder adenocarcinoma, n (\%) & $1(6.7 \%)$ \\
\hline Breast ductal carcinoma, n (\%) & $1(6.7 \%)$ \\
\hline Colon adenocarcinoma, n (\%) & $1(6.7 \%)$ \\
\hline Gastric adenocarcinoma, n (\%) & $1(6.7 \%)$ \\
\hline \multicolumn{2}{|c|}{ Stage of Cancer } \\
\hline Stage III, n (\%) & $1(6.7 \%)$ \\
\hline Stage IV, n (\%) & $14(93.3 \%)$ \\
\hline \multicolumn{2}{|c|}{ Malnutrition or Cancer Cachexia } \\
\hline Yes, n (\%) & $10(66.7 \%)$ \\
\hline No, $\mathrm{n}(\%)$ & $5(33.3 \%)$ \\
\hline \multicolumn{2}{|c|}{ Chemotherapy within 30 days } \\
\hline
\end{tabular}




\section{Cancer Therapy \& Oncology International Journal}

\begin{tabular}{|c|c|c|}
\hline Yes, n (\%) & $11(73.3 \%)$ \\
\hline No, n (\%) & $4(26.7 \%)$ \\
\hline \multicolumn{2}{|c|}{ Biliary Drainage and/or Prosthesis } \\
\hline Yes, n (\%) & $7(46.7 \%)$ \\
\hline No, n (\%) & 8(53.3\%) \\
\hline \multicolumn{2}{|c|}{ Grade 3-4 Neutropenia } \\
\hline Yes, n (\%) & & $2(13.3 \%)$ \\
\hline No, n (\%) & $13(86.7 \%)$ \\
\hline
\end{tabular}

\section{Primary and Secondary end-points}

At the time of the study analysis, 10 patients (66.7\%) had died and 5 patients (33.3\%) were still alive. In the entire population of the study $(\mathrm{n}=15)$, with a median follow-up of 18 months (95\% CI: 13-21 months), median OS was 6 months (95\% CI: 1-12 months); and 12 -month and 18-month os probability was $36 \%$ and $27 \%$, respectively (Figure 1). In our study, mean hospital stay of the cancer patients with liver abscesses was 30.5 days (95\% CI: 20.340.7 days; range: 6-65 days). Probable risk factors for developing liver abscesses in cancer patients identified in our study might be: a) the presence of a bilio-pancreatic cancer, which was present in $80 \%(12 / 15)$ of the patients, especially pancreatic cancer $(53.3 \%)$; b) administration of chemotherapy within 30 days before liver abscesses diagnosis, since $73.3 \%(11 / 15)$ of the patients in our study were treated with chemotherapy; c) malnutrition or tumor cachexia, which was present in $66.7 \%$ (10/15) of the patients; and d) biliary drainage and/or biliary prosthesis, which was present in $46.7 \%(7 / 15)$ of the study patients.

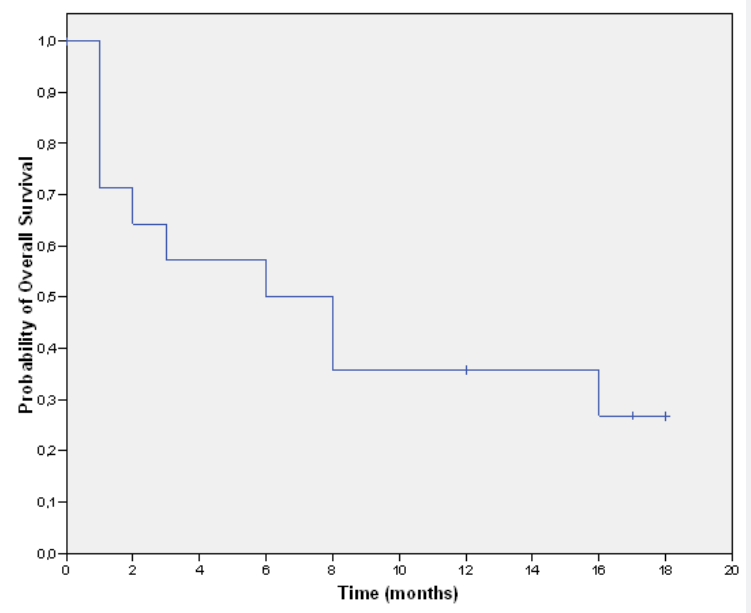

Figure 1: Kaplan-Meier estimates of Overall Survival (OS).

In the entire population of the study ( $n=15)$, with a median follow-up of 18 months (95\% Cl: 13-21 months), median OS was 6 months (95\% Cl: $1-12$ months); and 12 -month and 18 -month OS probability was $36 \%$ and $27 \%$, respectively

In this study, only 3 out of 15 (20\%) patients were treated with high doses of corticosteroids (prednisone $>20 \mathrm{mg}$ per day or equivalent doses) within 30 days prior to the diagnosis of liver abscesses. Grade 3-4 neutropenia was only presentin $13.3 \%$ (2/15) of patients at the time of liver abscesses diagnosis $[3,4]$. Thus, they do not seem to be a risk factor for developing liver abscesses for cancer patients. Eight patients (53.3\%) had bacteremia: in five patients (33.3\%) blood cultures were positive for Escherichia coli, in two patients $(13.3 \%)$ were positive for Enterococcus faecium and in one patient (6.7\%) was positive for Bacteroides fragilis [5]. Percutaneous drainage of the liver abscesses was performed in
$53.3 \%(8 / 15)$ of the patients, and all patients were treated with broad-spectrum antibiotics: $46.7 \%$ (7/15) with meropenem, $40 \%(6 / 15)$ with piperacillin-tazobactam, and $13.3 \%$ (2/15) with other antibiotic combinations.

\section{Discussion}

Liver abscesses are uncommon, but they are associated with an overall hospital mortality rate of $6-10 \%$ in patients without cancer [1]. However, the relevance of liver abscesses in patients with cancer in terms of survival and length of hospital stay is unknown [6,7]. Thus, we conducted this study in order to 


\section{Cancer Therapy \& Oncology International Journal}

determine if the diagnosis of liver abscesses would be associated with worse outcome and longer hospital stay in cancer patients.

In our study, the incidence of liver abscesses was low in hospitalized cancer patients $(<2 \%)$. However, liver abscesses diagnosis in cancer patients, in this study, were associated with a long hospitalization, with a mean hospital stay of 30.5 days (95\% CI: 20.3-40.7 days). The diagnosis of liver abscesses in this group of patients was also associated with poor outcome [8-11]. In the entire study population, median OS was only 6 months $(95 \% \mathrm{CI}$ : 1-12 months); and the OS probability at 18 months was only $27 \%$. This poor outcome, at least in part, may be due to the presence of advanced tumors with bad prognosis $(80 \%$ of patients had a bilio-pacreatic cancer with stage III-IV) and the malnutrition status (present in two third of the patients), since both of them are associated with reduced survival in cancer patients.

In our study, we identified some risk factors for developing liver abscesses in cancer patients, such as the presence of biliopancreatic cancers ( $80 \%$ of the patients), administration of chemotherapy within 30 days before liver abscesses diagnosis ( $73 \%$ of the patients), malnutrition or tumor cachexia $(67 \%$ of the patients), and biliary drainage and/or biliary prosthesis (47\% of the patients). Neither high doses of corticosteroids (prednisone $>20 \mathrm{mg}$ per day or equivalent doses) within 30 days prior to the diagnosis of liver abscesses (present in $20 \%$ of patients), nor grade 3-4 neutropenia (present only in $13 \%$ of patients) seem to be a risk factor for developing liver abscesses for cancer patients in this study. Half of patients had positive blood cultures (bacteremia). The most common isolated microorganism was E.coli (33\%), followed by E. faecium (13\%). All patients were treated with broad-spectrum antibiotics ( $47 \%$ with meropenem and $40 \%$ with piperacillin-tazobactam), and percutaneous drainage of the liver abscess was performed in $53 \%$ of the patients.

In summary, although liver abscesses are uncommon, in our study they were associated with a prolonged hospitalization and poor outcome in cancer patients. Risk factors for developing liver abscesses in cancer patients might be the presence of a bilio-pancreatic cancer, chemotherapy administration, severe malnutrition and presence of biliary drainage or prosthesis.

\section{Conflict of Interest}

The authors declare that they have no conflict of interest.

\section{References}

1. Lee KT, Wong SR, Sheen PC (2001) Pyogenic liver abscess: an audit of 10 years' experience and analysis of risk factors. Dig Surg 18(6): 459465.

2. Li YD, Lamano JB, Lamano JB, Quaggin-Smith J, Veliceasa D, et al. (2019) Tumor-induced peripheral immunosuppression promotes brain metastasis in patients with non-small cell lung cancer. Cancer Immunol Immunother 68(9): 1501-1513.

3. Patel K, West HJ (2017) Febrile Neutropenia. JAMA Oncol 3(12): 1751.

4. Phillips B, Morgan JE, Haeusler GM, Riley RD (2019) Individual participant data validation of the PICNICC prediction model for febrile neutropenia. Arch Dis Child.

5. Qu K, Liu C, Wang ZX, Tian F, Wei JC, et al. (2012) Pyogenic liver abscesses associated with nonmetastatic colorectal cancers: an increasing problem in Eastern Asia. World J Gastroenterol 18(23): 2948-2955.

6. Mohan BP, Meyyur-Aravamudan V, Khan SR, Chandan S, Ponnada S, et. al. (2019) Prevalence of colorectal cancer in cryptogenic pyogenic liver abscess patients. Do they need screening colonoscopy? A systematic review and meta-analysis. Dig Liver Dis 51(12): 1641-1645.

7. Jia Z, Tu J, Cao C, Wang W, Zhou W, et al. (2018) Liver abscess following transarterial chemoembolization for the treatment of hepatocellular carcinoma: A retrospective analysis of 23 cases. J Cancer Res Ther 14: S628-S633.

8. Chan KKW, Guo H, Cheng S, Beca JM, Redmond-Misner R, et al. (2019) Real-world outcomes of FOLFIRINOX vs gemcitabine and nab-paclitaxel in advanced pancreatic cancer: A population-based propensity score-weighted analysis. Cancer Med 9(1): 160-169.

9. Conroy T, Desseigne F, Ychou M, Bouché O, Guimbaud R, et al. (2011) Folfirinox versus gemcitabine for metastatic pancreatic cancer. $\mathrm{N}$ Engl J Med 364(19): 1817-1825.

10. Valle J, Wasan H, Palmer DH, David Cunningham, Alan Anthoney et al (2010) Cisplatin plus gemcitabine versus gemcitabine for biliary tract cancer. N Engl J Med 362: 1273-1281.

11. Loumaye A, Thissen JP (2017) Biomarkers of cancer cachexia. Clin Biochem 50(18): 1281-1288. 
This work is licensed under Creative Commons Attribution 4.0 License DOI: 10.19080/CTOIJ.2020.15.555914

\section{Your next submission with Juniper Publishers} will reach you the below assets

- Quality Editorial service

- Swift Peer Review

- Reprints availability

- E-prints Service

- Manuscript Podcast for convenient understanding

- Global attainment for your research

- Manuscript accessibility in different formats

( Pdf, E-pub, Full Text, Audio)

- Unceasing customer service

Track the below URL for one-step submission https://juniperpublishers.com/online-submission.php 